\title{
Comparative Study of Transabdominal Ultrasonography and Multidetector Row Computed Tomography in Preoperative Staging of Gastric Carcinoma
}

\author{
Sagar M.P ${ }^{1}$, Indira Narayanaswamy ${ }^{2}$, Ramprakash H.V ${ }^{3}$ \\ ${ }^{1}$ Assistant Professor, ${ }^{2}$ Professor, ${ }^{3}$ Professor and HOD, Vydehi Institute of Medical Sciences and Research Centre, Epip Area, \\ Whitefield, Bangalore-66, India
}

Corresponding author: Dr Indira Narayanaswamy, 2066, 16th C Main, HAL II Stage, Bangalore 560008, India

DOI: http://dx.doi.org/10.21276/ijcmsr.2018.3.4.27

How to cite this article: Sagar M.P, Indira Narayanaswamy, Ramprakash H.V. Comparative study of transabdominal ultrasonography and multidetector row computed tomography in preoperative staging of gastric carcinoma. International Journal of Contemporary Medicine Surgery and Radiology. 2018;3(4):D118-D121.

\section{A B S T R A C T}

Introduction: Gastric cancer is one of the most common malignant tumours in the digestive tract and is the second most common cause of cancer-related death worldwide. The incidence in India is 7-8 cases per 100,000 persons per year and it is the most common cancer in males in Chennai. The aim of the present study was to compare Ultrasonography (USG) and MDCT findings in the detection and staging of gastric carcinoma by correlating with histopathology.

Material and methods: Data were collected from 30 cases of clinically suspected/proven cases of gastric carcinoma that were referred to the Department of Radiodiagnosis for Ultrasonography and MDCT. Ultrasound examination was performed using a real time ultrasound with curvilinear transducer of 2-5 MHz and linear transducer of 7-10 MHz with ingestion of boiled cooled water. Plain and contrast MDCT study was performed in all the cases. The comparative study of ultrasonography and MDCT in diagnosing and staging of gastric carcinoma were analysed.

Results: The accuracy in detection of gastric carcinoma by USG was $80 \%$ (24/30 cases) and MDCT was $87 \%$ (26/30 cases), with $\mathrm{p}$ value of $<0.01$ which is significant, which shows CT is better modality for diagnosing gastric carcinoma. For T staging, the efficacy of MDCT and USG was $90 \%$ (27/30 cases) and 80\% (24/30 cases) respectively. For nodal status, the accuracy of CT is $73.3 \%(22 / 30$ cases) and USG is $63.3 \%$ (19/30 cases). For distant metastasis, accuracy was same with USG and CT (70\%, 27/30 cases). But when both the modalities are combined, the efficacy improves for diagnosing and staging gastric carcinoma by $93 \%$ with $\mathrm{P}<0.05$. The anastomosis degree of the gastric carcinoma between enhanced CT and ultrasonography was $\mathrm{k}=0.404$

Conclusion: Overall USG and Multi-detector row CT with combined water and air distention can improve the accuracy of preoperative staging of gastric cancer. Comprehensive imaging of US and MDCT is helpful to improve the accuracy of detection and staging of gastric carcinoma.

Key words: Gastric Carcinoma, Ultrasonography, MDCT, Accuracy, Detection, Staging

\section{INTRODUCTION}

Gastric cancer is one of the most common malignant tumors in the digestive tract and is the second most common cause of cancer-related death worldwide. ${ }^{1}$ The incidence in India is $7-8$ cases per 100,000 persons per year and it is the most common cancer in males in Chennai. ${ }^{2}$

The survival rates of patients with gastric cancers are closely related to gastric wall invasion ( $\mathrm{T}$ staging), lymph node metastasis ( $\mathrm{N}$ staging), and distant organ metastasis of gastric tumours ( $\mathrm{M}$ staging). If stomach cancer is accurately diagnosed at an early stage, followed by a timely surgery, there is a good chance for patients to be cured and live for a long time. Even for patients with advanced gastric cancer, obtaining an accurate diagnosis and precise staging, the survival time can be extended by chemotherapy or neoadjuvant chemotherapy. ${ }^{3}$
Transabdominal ultrasound is the least invasive and cost effective imaging technique available for evaluating gastric cancer. It has recently been shown that dedicated transabdominal ultrasound performed after ingestion of boiled cooled water and of a hypotonic agent along with the use of broad band transducers provides detailed and unique evaluation of the stomach. ${ }^{4}$

Multidetector CT involves thin-section collimation of the stomach allowing high-quality MPR (Multiplanar Reformations) images which dramatically improves the visualization of fine anatomic details of the gastric wall, adjacent structural involvement, lymph node status and metastatic lesions. ${ }^{5}$

The purpose of this study was to compare ultrasonography and MDCT in detection of gastric carcinoma. To compare ultrasonography and MDCT in staging of gastric carcinoma with histopathology as reference. 


\section{MATERIAL AND METHODS}

Patients with clinical suspicion and with diagnosis of gastric cancer, referred to Department of Radio diagnosis, for cross sectional imaging were recruited for study. Thirty patients were examined by both USG and MDCT and selected for the study. Patients with clinical suspicion and diagnosed to have gastric malignancy were included. Pregnant women and patients allergic to contrast media were excluded.

\section{USG Imaging protocol}

The gray scale sonographic examination using 2-5 $\mathrm{MHz}$ multi-frequency transducer in PHILIPS HD 15 ultrasound machine. Patients should keep an empty stomach before ultrasound examination. Five minutes before the examination, the patient is asked to drink about 500 to $800 \mathrm{~mL}$ of boiled cooled water. Pre-medication infusion of $20 \mathrm{mg}$ Buscopan is given. Scanning is done in longitudinal, transverse and left sided subcostal oblique views. During scanning, if lesions are in the cardia, the supine position should be used; if lesions are in the gastric fundus, gastric body, or the gastric antrum, the right lateral position is used. When imaging is not satisfactory, the left lateral, half-sitting, sitting, and standing position could be used.

\section{CT imaging protocol}

CT scan was obtained on a GE Lightspeed Xtra 16 slice multi detector scanner (GE Medical Systems, Milwaukee, United States). Plain CT study from the dome of the diaphragm to the pubic symphysis were obtained with a scan exposure time of 9.48 seconds, slice thickness of $5 \mathrm{~mm}$, pitch 1.375:1, speed 27.50 ( $\mathrm{mm} /$ rotation). Subsequently, contrast enhanced CT was performed using automated pressure injector and injecting bolus of $70 \mathrm{ml}$ of intravenous contrast of Iohexol at the rate of $3.5 \mathrm{ml} /$ second. All patients received oral contrast. Contrast enhanced images were acquired at 20 seconds for arterial phase, 65 seconds for venous phase and 6 minutes for delayed after the injection of IV contrast. All images were acquired during inspiration with $120 \mathrm{kV}, 250-300 \mathrm{~mA}$. The venous phase images were then reconstructed using inbuilt GE image works software to $1.25 \mathrm{~mm}$ thickness and the examination was pushed to Advanced Workstation console for viewing and reporting. The images were then displayed in $512 \times 512$ matrixes in two high resolution medical grade monitor. Images were analysed with Multiplanar Reconstruction (MPR), bone window and soft tissue algorithm.

Gastric carcinoma was staged preoperatively with USG and MDCT, and postoperatively with histopathologic examination (HPE). Tumour staging was evaluated using the Tumor-Node-Metastasis (TNM) staging and Japanese classification. ${ }^{6}$ The results from the imaging modalities were compared with the postoperative histopathological outcomes (Image 1,2).

\section{STATISTICAL ANALYSIS}

Data analysis was performed with SPSS statistical software. With use of $2 \times 2$ contingency table, descriptive statistics were obtained. Cross tabs with chi square test analysis was done for comparison of parameters of different modality. Statistically significant differences were indicated with $\mathrm{p}$ less than 0.01 .

\section{RESULTS}

Thirty patients ranging between 30 and 68 years with mean age of $49.87 \pm 11.22$ years. $24(80 \%)$ patients were males and $6(20 \%)$ were females. Pain abdomen and vomiting were the most common symptoms accounting for 13 cases (43.3\%). Greater and lesser curvature were more commonly involved accounting for $16 \%$ (5 cases).

Diagnostic accuracy of the ultrasonography in detection of the gastric carcinoma was found to be $80 \%$, accuracy in detection by MDCT was found in $87 \%$. In present study, CT had 87\% efficacy, whereas USG had 80\% efficacy with $\mathrm{P}$ value $<0.01$ which was significant, which shows $\mathrm{CT}$ is better modality for diagnosing gastric carcinoma (graph-1, 2). Tumour extension was clearly identified by ultrasound in $80 \%$ of cases and $90 \%$ of cases on MDCT. For nodal status, the accuracy of CT is $73.3 \%$ and USG is $63.3 \%$. For distant metastasis, accuracy was same with USG and CT. The accuracy of $\mathrm{T}$ staging in gastric carcinoma by both enhanced CT and ultrasound was higher than that either by enhanced CT or by ultrasound, respectively with $\mathrm{P}<0.05$. The anastomosis degree of the gastric carcinoma between enhanced CT and ultrasonography was $\kappa=0.404$. Hence, when both the modalities are combined, the efficacy

\section{USG VS CT}

Diagnosed $\square$ Un diagnosed

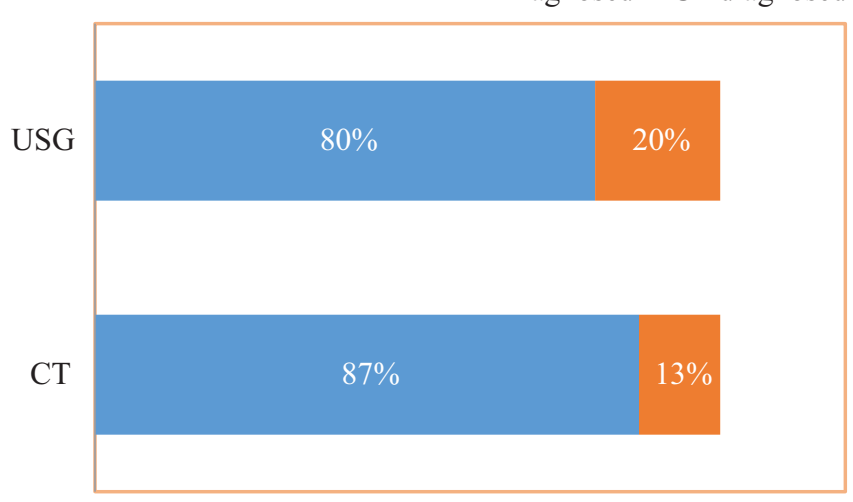

USG-Carcinoma present in 24 cases.

MDCT-Carcinoma present in 26 cases

Graph-1: showing diagnosed and undiagnosed percentage of CT vs USG

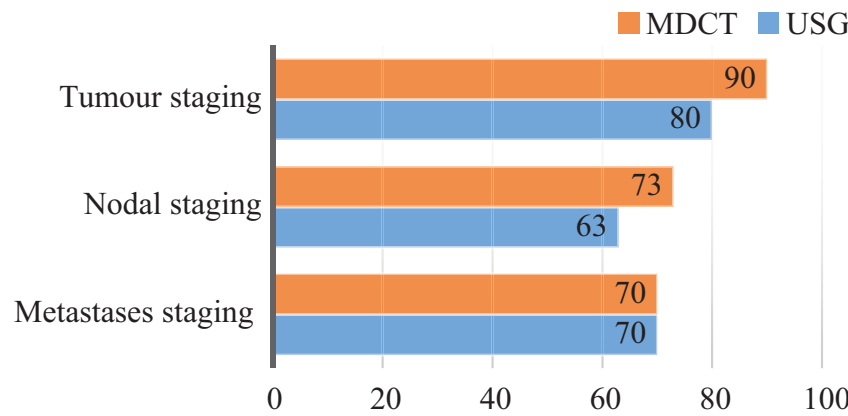

T staging CT 27 cases, US 24 cases $\mathrm{N}$ staging CT 22 cases, USG 19 cases Metastases 27 cases

Graph-2: Showing the percentage of tumour, nodal and metastases extension on MDCT and USG 


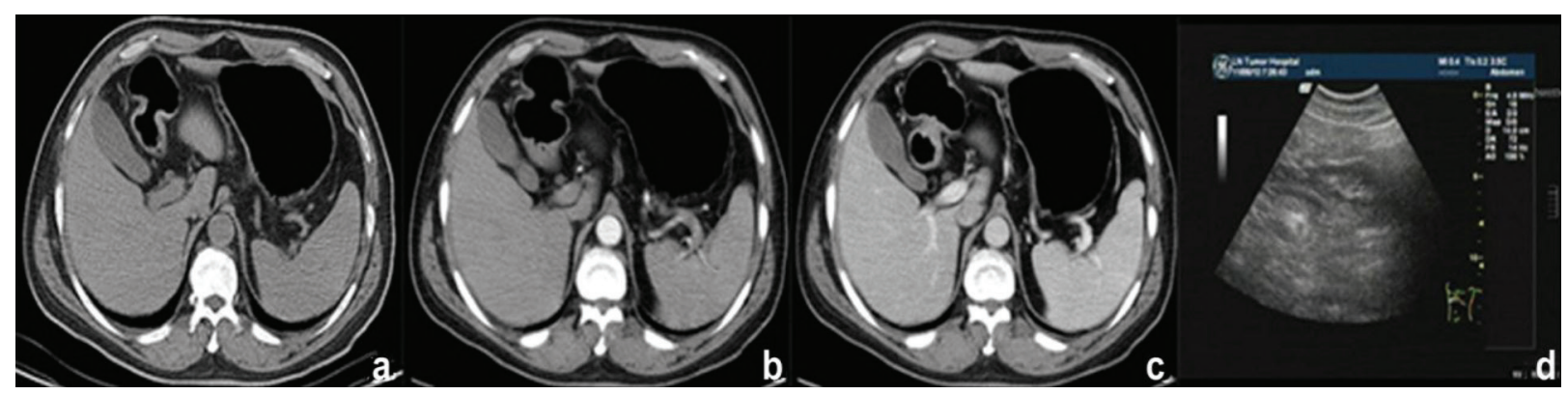

Image-1: T2 gastric cancer in gastric antrum. (a-c) Enhanced CT scanning showed a clear gap surrounding the fat and smooth serosa. (d) The ultrasound imaging. Visible lesion invading the muscle layer.

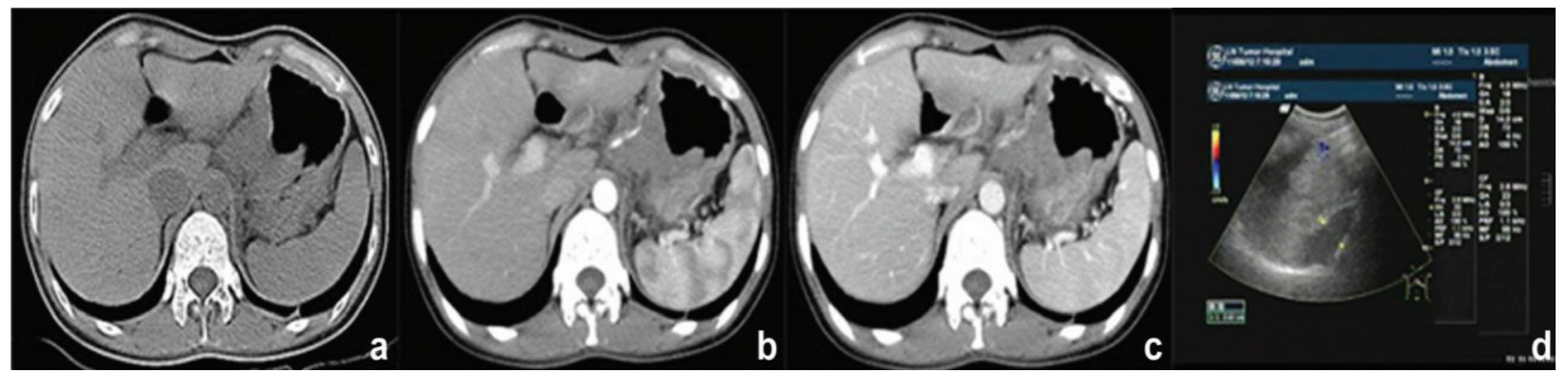

Image-2: T4 gastric cancer in cardia and gastric fundus. (a-c) CT scanning. The gap around fat tissues disappeared, and the lesion invaded other structures such as the tail of the pancreas, left gastric artery. (d) Ultrasound imaging. It was visible that lesion invading the surrounding organs.

improves for both diagnosing and staging gastric carcinoma.

\section{DISCUSSION}

Accurate preoperative diagnosis and staging of patients with gastric cancer is essential for optimal treatment. ${ }^{3}$ The methods used for evaluating the gastric cancer are upper gastrointestinal series (barium meal) and conventional endoscopy. Both these modalities fail to identify the adjacent structure involvement.

Upper gastrointestinal endoscopy is the preferred technique for detection of GC which is also useful in obtaining histological confirmation of suspicious gastric lesions. However, up to $6.7 \%$ of GCs may be missed when an endoscopy shows no initial cancer finding. Endoscopic ultrasound is available only in select centres. ${ }^{7}$

Ultrasonography (USG) and Multidetector Computed Tomography (CT) with Multiplanar Reformation (MPR) gives both physiological as well as anatomical description of the site and extension of lesion within the lumen, as well as beyond the lumen., ${ }^{4,5}$ The other modalities which are used are Magnetic Resonance Imaging (MRI) and Positron Emission Tomography (PET). These examinations are effective to varying degrees and are limited in assessing the site and extension of disease. ${ }^{8}$ Transabdominal ultrasound is the least invasive and cost effective imaging technique available for evaluating gastric cancer. It has recently been shown that dedicated transabdominal ultrasound performed after ingestion of boiled cooled water and of a hypotonic agent along with the use of broad band transducers provides detailed and unique evaluation of the stomach. ${ }^{4}$

Multidetector CT involves thin-section collimation of the stomach allowing high-quality MPR (Multiplanar Reformations) images which dramatically improves the visualization of fine anatomic details of the gastric wall, adjacent structural involvement, lymph node status and metastatic lesions. ${ }^{9}$

In present study, CT showed $87 \%$ efficacy, whereas USG showed $80 \%$ efficacy with $\mathrm{P}$ value $<0.01$ which is significant, which shows CT is better modality for diagnosing gastric carcinoma. For T staging, the efficacy of MDCT and USG was $90 \%$ (27/30 cases) and 80\% (24/30 cases) respectively. For nodal status, the accuracy of CT is $73.3 \%$ (22/30 cases) and USG is $63.3 \%$ ( $19 / 30$ cases). For distant metastasis, accuracy was same with USG and CT with accuracy of $70 \%$ (27/30 cases). The accuracy of $\mathrm{T}$ staging in gastric carcinoma by both enhanced CT and ultrasound was higher than that either by enhanced CT or by ultrasound by $93 \%$, with $\mathrm{P}<0.05$. The anastomosis degree of the gastric carcinoma between enhanced CT and ultrasonography was $\kappa=0.404$. Hence, when both the modalities are combined, the efficacy improves for both diagnosing and staging gastric carcinoma. This is seen in consensus with studies done by Venkat et al and San Sui. ${ }^{4,9}$

The performance of ultrasonography is found to be better in $\mathrm{T} 1$ and T2 staging and decreases in T3 and T4, due to the lesions being far from the ultrasound probe, resulting in a lower spatial resolution. While T3 to T4 gastric T staging is the advantage of enhanced MDCT scanning.

T1 and T2 staging is inferior by MDCT as the resolution in to the mucosa and submucosa is difficult in some cases. When both the modalities (oral echo-type filling agent ultrasound and MDCT) are combined there is improved results in both diagnosing as well as staging of gastric carcinoma.

\section{CONCLUSION}

Multidetector computed tomography (MDCT) today is 
gold standard in establishing the extent of gastric carcinoma. However, Ultrasonography (US) continues to be the simple evaluation technique which is universally available and with proper application it could yield diagnostic value on par with MDCT. A global view of the MDCT and US results are required to obtain an accurate assessment of gastric wall invasion ( $\mathrm{T}$ staging), lymph node metastasis ( $\mathrm{N}$ staging), and distant organ metastasis ( $\mathrm{M}$ staging) of gastric tumours, as both modalities exhibited advantages in TNM staging. We suggest that combined US and MDCT be performed for diagnostic and preoperative evaluation of gastric cancer.

\section{REFERENCES}

1. Ferlay J, Shin HR, Bray F, Forman D, Mathers C et al. Estimates of worldwide burden of cancer in 2008: GLOBOCAN 2008. Int J Cancer 2010; 127(1): 28932917.

2. Cancer Incidence and Mortality in greater Mumbai, 1994. Mumbai, India: Report of Bombay Cancer Registry; 1998. Bombay Cancer Registry.

3. Cunningham D, Allum WH, Stenning SP, et al. Perioperative chemotherapy versus surgery alone for resectable gastroesophageal cancer. N Engl J Med.

4. Venkataraman I, Rao HK, Singh P, Elangovan S, Kate $\mathrm{V}$. The efficacy of hydrogastric sonography and Spiral Computed Tomography in staging of gastric carcinoma -a comparative. J Clin Ultrasound 2010;88(9):480-5.

5. Blackshaw GR, Stephens MR, Lewis WG, et al. Progressive CT system technology and experience improve the perceived preoperative stage of gastric cancer. Gastric Cancer. 2005; 8(3):29-34.

6. Edge SB, Compton CC. The American Joint Committee on Cancer: the 7th edition of the AJCC cancer staging manual and the future of TNM. Ann Surg Oncol. 2010; 17(1):1471-1474.

7. Linlin Zhu et al. Early Gastric Cancer: Current Advances of Endoscopic Diagnosis and Treatment. Gastroenterology Research and Practice. Volume 2016 (2016)

8. Chen-Xi Wu and Zhao-Hui Zhu. Diagnosis and evaluation of gastric cancer by positron emission tomography. (World J Gastroenterol. 2014 Apr 28; 20(16): 4574-4585.

9. Tao Yu et al. Prediction of $\mathrm{T}$ stage in gastric carcinoma by enhanced CT and oral contrast-enhanced. World Journal of Surgical Oncology 2015;13(3):184.

Source of Support: Nil; Conflict of Interest: None

Submitted: 22-11-2018; Accepted: 20-12-2018; Published online: 29-12-2018 